\title{
Intoxicación Neonatal por Sulfato de Magnesio: Caso clínico
}

\author{
MAGDALENA CRUZ O. ${ }^{1}$, ADRIANA DOREN V. ${ }^{1}$, ROCÍO FERNÁNDEZ B. ${ }^{1}$, \\ JOSÉ ANTONIO SALINAS T. ${ }^{2}$, SOLEDAD URZÚA B. ${ }^{1}$, JOSÉ LUIS TAPIA I. ${ }^{1}$ \\ 1. Unidad de Neonatología, Departamento Pediatría. \\ 2. Unidad de Neonatología, Becado Neonatología. \\ Escuela de Medicina, Pontificia Universidad Católica de Chile.
}

\begin{abstract}
Magnesium Neonatal Intoxication: A Case Review

Magnesium Sulfate is currently the treatment of choice for severe preeclampsia and eclampsia. Since it crosses the placenta, magnesium can reach high levels in fetal plasma, ocassionally reaching levels higher than maternal plasma. Objective: To analyze a 35 weeks gestational age newborn, who presented hypermagnesemia due to maternal treatment with magnesium sulfate (in routine dosage) indicated for severe preeclampsia. Clinical Case: The mother was infused with $\mathrm{MgSO} 4$ for 17 hours until pregnancy was interrupted because of fetal monitoring. The baby weighed 2,620 grams, was depressed, hypotonic, cyanotic and without respiratory effort and only partially responded to initial resucitation with positive pressure ventilation. The infant was admitted to the Neonatal Intensive Care Unit for monitoring, support and management. High levels of plasma $\mathrm{MgSO} 4$ were found $(4.7 \mathrm{mg} / \mathrm{dl})$, compatible with the diagnosis of hypermagnesemia. Symptoms and signs slowly disappeared, and the child was discharged after 10 days of hospitalization. Conclusion: We present the clinical case of a newborn with a history of maternal use of magnesium sulfate, who presented hypermagnesemia characterized by neonatal depression, hypotonia, central apnea with oxygen requirement and who progressively normalized in a 10 day period.

(Key words: Magnesium sulfate, infant, newborn, preeclampsia).

Rev Chil Pediatr 2009; 80 (3): 261-266
\end{abstract}

\section{RESUMEN}

El Sulfato de Magnesio es actualmente el medicamento de elección para el tratamiento de la embarazada con preeclampsia severa y eclampsia. Dado que atraviesa la placenta, alcanza elevados niveles en el plasma fetal, pudiendo en ocasiones superar la concentración plasmática materna. Objetivo: Analizar la evolución de un neonato de 36 semanas de edad gestacional que cursó con cuadro de hipermagnesemia secundario a tratamiento materno con sulfato de magnesio en dosis habituales, indicado como terapia de una

Trabajo recibido el 13 de noviembre de 2008, devuelto para corregir el 12 de enero de 2009, segunda versión el 03 de febrero de 2009, aceptado para publicación el 23 de marzo de 2009.

Correspondencia a:

Dr. José Luis Tapia I.

E-mail: jlta@med.puc.cl 
preeclampsia severa. Caso clínico: Madre recibe infusión de MgSO4 por un total de 17 hrs antes de decidirse la interrupción del embarazo por vía alta debido a un registro sospechoso. Se obtuvo recién nacido de 2620 gramos, deprimido, sin esfuerzo respiratorio, hipotónico y cianótico, que respondió parcialmente a la reanimación inicial con ventilación a presión positiva. Se decidió ingreso a Cuidados Intensivos de la Unidad de Neonatología para monitorización, soporte y manejo. Se detectaron niveles plasmáticos elevados de $\mathrm{MgSO} 4$ de 4,7 mg/dL compatibles con el diagnóstico de Hipermagnesemia. La signología fue disminuyendo progresivamente, decidiéndose el alta luego de 10 días de hospitalización. Conclusión: Se presenta caso clínico de un recién nacido, con antecedente de sulfato de magnesio administrado a la madre y que evoluciona con un cuadro de hipermagnesemia caracterizado por depresión neonatal, apneas centrales, requerimientos de oxígeno e hipotonía, los cuales van normalizándose progresivamente en el curso de 10 días.

(Palabras clave: Hipermagnesemia, recién nacido, preeclampsia).

Rev Chil Pediatr 2009; 80 (3): 261-266

\section{Introducción}

El sulfato de magnesio es actualmente la terapia de elección para el manejo de pacientes con preeclampsia severa. Debido a su frecuente uso en obstetricia surge la necesidad de conocer su real indicación, dosis y efectos tantos terapéuticos como desfavorables.

Tras la exposición de un caso clínico referente a un recién nacido con intoxicación por Sulfato de Magnesio subsecuente al tratamiento materno con este fármaco debido a preclampsia severa; realizamos una revisión de la literatura disponible sobre las principales manifestaciones maternas y neonatales descritas como consecuencia de la intoxicación por este fármaco. Se incluye brevemente la evidencia existente acerca del manejo de los recién nacidos que sufren de este cuadro clínico.

\section{Caso clínico}

Recién nacido de pretérmino de 36 semanas, de sexo masculino, hijo de madre primigesta de 21 años sin antecedentes mórbidos, con embarazo bien controlado, que cursó con Síndrome Hipertensivo del Embarazo detectado a las $35+3$ semanas de edad gestacional.

Se derivó desde atención primaria a nuestro centro por hallazgo de cifras tensionales elevadas en dos oportunidades (140/100 mmHg y 150/100 mmHg), asintomática. En Servicio Urgencia de Maternidad se constató presión arterial de $150 / 100 \mathrm{mmHg}$, albuminuria cualitativa +++ en 2 tomas consecutivas separadas por 6 horas, y examen físico normal. Se ingresó con diagnóstico de Síndrome Hipertensivo del Embarazo con posible Preeclampsia Moderada, iniciándose tratamiento con alfa metildopa 500 mg cada 8 horas por vía oral. La evaluación de unidad fetoplacentaria y los exámenes de severidad fueron normales. La proteinuria de 24 horas fue de 7,307 gramos razón por la cual se diagnosticó Preeclampsia Severa, se inició terapia con sulfato de magnesio $\left(\mathrm{MgSO}_{4}\right)$ en dosis habituales ( 5 gr en bolo endovenoso y luego 1 gr por hora a mantención por bomba de infusión continua) y se decidió interrupción del embarazo. Se administró misoprostol vía vaginal en 3 oportunidades, una ampolla de ketorolaco para manejo del dolor y profilaxis con ampicilina endovenosa por antecedente de colonización materna desconocida por Streptococcus Grupo B. Debido a falta de progresión del trabajo de parto asociada a registro sospechoso se decidió interrupción vía cesárea a las 12 horas de iniciada la inducción, habiendo recibido $\mathrm{MgSO}_{4}$ por un total de $17 \mathrm{hrs}$ (22 gr en total de $\left.\mathrm{MgSO}_{4}\right)$. La cirugía se realizó sin incidentes bajo anestesia regional, observándose líquido amniótico claro sin distocia de cordón.

Se obtuvo recién nacido de 2620 gramos y $47,5 \mathrm{~cm}$ de talla, adecuado para la edad gestacional. Nace deprimido, sin esfuerzo respiratorio, hipotónico y cianótico, con un Apgar al minuto de 2 (frecuencia cardíaca: 2), y luego de 6 y 7 a $\operatorname{los} 5$ y 10 minutos respectivamente. 
Requirió ventilación a presión positiva y oxígeno libre, sin llegar a la intubación. Dentro de los exámenes realizados destacó un $\mathrm{pH}$ arterial de recién nacido, obtenido minutos después del parto, de 7,12, $\mathrm{pCO}_{2}$ de 58,6 $\mathrm{mmHg}, \mathrm{HCO}_{3}$ de $19,4 \mathrm{mg} / \mathrm{dL}$ y BE de -10 . Luego de la reanimación inicial, el neonato persistió con respiración irregular, hipotónico, con requerimientos de $\mathrm{O}_{2}$ de $22 \%$, orinando de forma adecuada (diuresis primer día de vida: $2,87 \mathrm{cc} / \mathrm{kilo} / \mathrm{hora}$ ). Se decidió ingreso a Cuidados Intensivos de la Unidad de Neonatología para monitorización y manejo, con diagnóstico de depresión respiratoria.

Ante la sospecha de una intoxicación por $\mathrm{MgSO}_{4}$ se midieron niveles plasmáticos al ingreso, que revelaron un valor elevado de 4,7 $\mathrm{mg} / \mathrm{dL}$ (valor normal: $1,6-2,2 \mathrm{mg} / \mathrm{dL})^{1}$ compatible con el diagnóstico de hipermagnesemia. El recién nacido evolucionó con requerimientos de $\mathrm{O}_{2}$ por 24 horas y normalización de la acidosis a las 3 horas de vida. Presentó tres episodios de apneas centrales dentro de los primeros 4 días de vida asociados a desaturación (hasta $\mathrm{SatO}_{2}$ $65 \%$ ), hipoactividad, tono axial disminuido y reflejo de moro débil, que cedieron con estímulo manual, se atribuyeron a intoxicación por $\mathrm{MgSO}_{4}$ por lo que se administró una dosis de gluconato de calcio endovenoso de $0,2 \mathrm{mg} / \mathrm{kilo}$ de peso a las 48 horas del parto. A las 36 horas de vida se inició estímulo enteral progresivo con buena tolerancia, y a las 48 horas se trasladó a intermedio. Tanto la signología clínica como los niveles de sulfato de magnesio fueron disminuyendo progresivamente, con una última medición de éste al sexto día de $2,7 \mathrm{mg} / \mathrm{dL}$. Mantuvo una termorregulación y diuresis adecuadas durante toda su estadía, así como también nivel de creatinemia $(0,7 \mathrm{mg} / \mathrm{dl})$ y electrolitos plasmáticos dentro de rangos normales.

Dentro de la hospitalización destacó ictericia con una bilirrubina de $13,8 \mathrm{mg} / \mathrm{dL}$, por lo que se mantuvo en fototerapia por 24 horas con buena respuesta.

Dada buena evolución, sin apneas por una semana y con alimentación oral exclusiva (100 cc por kilo día) con buena tolerancia, se decidió el alta a los 10 días de vida, con control a las 48 horas ya que no recuperó el peso de nacimiento (2 495 gramos).

\section{Discusión}

El Sulfato de Magnesio $\left(\mathrm{MgSO}_{4}\right)$ es una droga que ha sido ampliamente usada en Obstetricia desde $1906^{2}$, siendo actualmente el tratamiento de elección en mujeres con preclampsia severa y eclampsia ${ }^{3}$ y que ha demostrado ser superior que otras terapias en la prevención de convulsiones en estas pacientes ${ }^{4-7}$. Si bien fue utilizado para prevenir el parto prematuro a partir de la década del sesenta, ha dejado de usarse como un agente tocolítico pues ha sido reemplazado por otras drogas más eficaces ${ }^{8,9}$.

Su componente principal, el Magnesio, es el catión bivalente más abundante en el líquido intracelular, donde participa en numerosas reacciones enzimáticas y en el metabolismo de hidratos de carbono, lípidos y proteínas. Sólo el $1 \%$ del Magnesio corporal total se encuentra en el extracelular, y es la concentración de éste la que es esencial para mantener una actividad neuromuscular normal, en conjunto con el Calcio. Ambos cationes compiten en el plasma por las mismas proteínas transportadoras, lo que produce un aumento del Magnesio iónico ante incrementos en el Calcio plasmático. Niveles estables de Magnesio sérico dependen principalmente de la ingesta diaria, de la presencia y relación con otros cationes como el Calcio, y de una adecuada excreción renal. La reserva inmediata disponible de Magnesio intercambiable, ante situaciones de baja ingesta, se obtiene del tejido óseo y muscular ${ }^{10}$.

Su mecanismo de acción no ha sido bien dilucidado, sin embargo, se han planteado numerosas hipótesis dentro de las cuales se incluyen la vasodilatación de la vasculatura cerebral, inhibición de la agregación plaquetaria, protección celular contra radicales libres, disminución de la liberación de acetilcolina (Ach) a nivel de la placa motora y antagonismo competitivo del receptor de glutamato (NMDA) ${ }^{11}$. Además, a nivel muscular, inhibiría la reacción entre la miosina y la ATPasa, provocando relajación en la placa motora. La irritabilidad neuromuscular depende por lo tanto, en gran medida del Magnesio, pero contribuyen a ésta otros cationes según la fórmula de Loeb. En ésta, la irritabilidad neuromuscular es directamente proporcional a las concentraciones de 
Sodio y Potasio, e inversamente proporcional a las de Calcio, Magnesio e Hidrogeniones. Se desprende de esta fórmula que la disminución tanto del magnesio como del calcio generarían una disminución de la actividad en la placa motora $^{10}$.

Se ha utilizado como forma de administración la vía intramuscular y endovenosa, siendo esta última la más segura ${ }^{12}$. Aún no existe consenso acerca de la dosificación óptima del $\mathrm{MgSO}_{4}$ ni en que momento debe iniciarse y terminarse la terapia, sin embargo, suele administrarse al inicio del trabajo de parto o de la inducción de éste ${ }^{13,14}$ manteniéndose por 24 horas después del parto dependiendo de la severidad del cuadro ${ }^{14}$. La dosis más frecuentemente utilizada consiste en un bolo de carga endovenoso de 4 a 6 gramos, seguida de una infusión continua de 1-3 gr/h/, $\mathrm{h}^{3,9,14-16}$. La Food and Drug Administration (FDA) recomienda una dosis máxima de 30 a 40 gramos en 24 horas. Dado que su mecanismo de excreción es exclusivamente renal la dosis debe ser ajustada en aquellas pacientes con insuficiencia renal ${ }^{9}$.

\section{Efectos maternos}

Los efectos maternos secundarios al uso de $\mathrm{MgSO}_{4}$ son típicamente dosis dependiente y de gran variedad en su forma de presentación y severidad clínica, observándose síntomas que van desde náuseas, vómitos, diaforesis, ileo paralítico, palpitaciones, y cefalea hasta debilidad muscular, disnea, hipotermia, hipotensión, dolor precordial y edema pulmonar, pudiendo llegar incluso al colapso cardiovascular y paro respiratorio ${ }^{9}$. Cabe destacar que el edema pulmonar se produce hasta en un 1 a $2 \%$ de las mujeres tratadas con sulfato de magnesio ${ }^{18}$.

Otros efectos observados con esta droga son hemorragia postparto por atonía uterina ${ }^{7}$, hipocalcemia debido a la supresión de la hormona paratiroidea (PTH), ileo paralítico y alteraciones en la capacidad de concentración ${ }^{19}$.

Por todo lo anterior la administración de $\mathrm{MgSO}_{4}$ debe ser monitorizada clínicamente mediante la observación seriada de tres parámetros: reflejos osteotendíneos, frecuencia respiratoria y diuresis. El rango terapéutico aceptado va de 4,8 a $8,4 \mathrm{mg} / \mathrm{dL}^{20}$, y al alcanzarse niveles mayores a éstos se observa el primer signo de intoxicación que corresponde a la disminución de los reflejos (con valores que oscilan entre 9 y $12 \mathrm{mg} / \mathrm{dL}$ ). Con niveles más elevados, entre 14 y $18 \mathrm{mg} / \mathrm{dL}(12$ a $14 \mathrm{mEq} / \mathrm{L})$ ocurre depresión respiratoria significativa; y si se llegase a concentraciones por sobre los 18 $\mathrm{mg} / \mathrm{dL}(15 \mathrm{mEq} / \mathrm{L})$ ocurre colapso cardiovascular $^{21}$.

\section{Efectos en el recién nacido}

$\mathrm{E} 1 \mathrm{MgSO}_{4}$ atraviesa rápidamente la placenta alcanzando niveles elevados tanto en el plasma fetal como en el líquido amniótico siendo éstos proporcionales a los encontrados en el suero materno ${ }^{22}$. Ante terapias prolongadas (mayor a 72 horas) la magnesemia fetal puede incluso superar la materna dada la inmadurez de su sistema excretor ${ }^{9}$.

En el recién nacido las manifestaciones clínicas de la hipermagnesemia presentan una amplia variabilidad y no se correlacionan de manera estricta con los niveles de $\mathrm{MgSO}_{4}$ como ocurre en la madre 9 . Se ha sugerido que esta discrepancia se debe a que en el feto el volumen de distribución cobra mayor importancia que el nivel plasmático en sí, siendo determinante el contenido intracelular de magnesio ${ }^{23}$.

Dentro de las principales manifestaciones destacan hipotonía, depresión respiratoria e hipotensión. La primera puede observarse en grado variable desde disminución de la succión, reflejos atenuados, llanto débil e incluso necesidad de ventilación a presión positiva. Desde el punto de vista cardiovascular se observa una disminución de la presión arterial y de la frecuencia cardíaca, no alterándose el gasto cardíaco debido al aumento compensatorio de la función ventricular ${ }^{24}$.

Se ha observado que el Sulfato de Magnesio no produce daño en la sustancia blanca ni aumenta la incidencia de hemorragia intraventricular ${ }^{25}$; de hecho se le ha atribuido una disminución en la frecuencia de leucomalacia y de parálisis cerebral en recién nacidos de bajo peso $^{26,27}$. Es por esto que incluso se ha estudiado el uso de sulfato de magnesio como medida neuroprotectora en partos de pretérmino, con buenos resultados neonatale ${ }^{28}$. La mortalidad neonatal como otras morbilidades hasta ahora 
no se han visto alteradas ${ }^{29}$, sin embargo, persiste el seguimiento de los pacientes involucrados en estos estudios.

Otros efectos analizados han sido la retinopatía del prematuro severa y convulsiones, no observándose relación entre éstas y el $\mathrm{MgSO}_{4}{ }^{30}$.

Si bien la hipermagnesemia se asocia a parálisis intestinal en adultos ${ }^{31}$, este efecto no se ha logrado establecer con claridad en los recién nacidos. En uno de los pocos estudios que se refiere al tema, la demora en el tránsito intestinal ocurrió en el $38 \%$ de los recién nacidos con hipermagnesemia versus un $15 \%$ del grupo control, diferencia que, sin embargo, no alcanzó a ser estadísticamente significativa ${ }^{32}$. Casos aislados de perforación intestinal, retardo en el tránsito intestinal y síndrome de tapón meconial han sido reportados por esta causa ${ }^{33-35}$.

\section{Manejo de la hipermagnesemia en el neonato}

El manejo del recién nacido depende de sus manifestaciones clínicas y la gravedad de éstas; en la mayoría de los casos los síntomas y signos mejoran en 48 a 72 horas con una terapia de soporte que incluye mantención de la ventilación y del equilibrio hidroelectrolítico ${ }^{36}$, junto con monitorización en una Unidad de Cuidados Especiales. Se debería realizar también un estricto control de parámetros de laboratorio tales como magnesio, electrolitos plasmáticos, calcio y fósforo, además de una monitorización de diuresis y tránsito intestinal.

Algunos neonatos pueden llegar a requerir ventilación asistida ${ }^{9} \mathrm{o}$ incluso resucitación cardiopulmonar avanzada con tubo endotraqueal para revertir los efectos sobre la dinámica ventilatoria, por lo que se recomienda una monitorización cardiorrespiratoria continua, para registrar y tratar rápidamente posibles apneas.

La alimentación de estos recién nacidos se realizaría de forma gradual, evaluando tolerancia y aparición de residuos a la aspiración gástrica. Sin embargo, no existen estudios que avalen esta práctica clínica.

El gluconato de calcio al 10\% se ha utilizado con el fin de antagonizar el efecto del magnesio a nivel del sistema nervioso. La dosis utilizada fluctúa entre 200 y $500 \mathrm{mg}$ endovenosos en cada administración $(100 \mathrm{mg} / \mathrm{kg})$, con moni- torización electrocardiográfica continua ${ }^{37} . \mathrm{Si}$ bien es una práctica ampliamente utilizada no existen aún estudios metodológicamente adecuados que respalden esta práctica y determinen la dosis ideal. Se han publicado algunas experiencias con el uso de este fármaco que muestran un efecto pasajero y eficacia par$\mathrm{cial}^{23,38}$. Casos extremos pueden requerir terapias más agresivas como hemodiálisis y exsanguíneo transfusión ${ }^{23,37}$.

\section{Conclusiones}

El sulfato de magnesio sigue siendo la terapia de elección para el manejo de pacientes con preeclampsia severa. Sin embargo, como podemos apreciar en esta revisión, su uso no está exento de complicaciones. Es por este motivo que los médicos que utilizan este fármaco deben conocer las indicaciones, dosis y posibles signos de intoxicación que se deben buscar tanto en embarazadas como en sus recién nacidos.

La sospecha clínica es de vital importancia al momento de pesquisar una posible intoxicación por sulfato, debido a que sus niveles plasmáticos no siempre se correlacionan fehacientemente con su expresión clínica. Es así como la medición rutinaria de niveles de magnesio en niños asintomáticos, hijos de madres hipertensas, no entrega información de utilidad, como tampoco la medición seriada para controlar niveles altos.

\section{Referencias}

1.- Cloherty J, Eichenwald E, Stark A: Manual of Neonatal Care. Editorial Lippincott Williams and Wilkins. Philadelphia 2004; 781.

2.- Chesley LC, Tepper I: Plasma levels of magnesium attained in magnesium sulphate therapy for preeclampsia and eclampsia. Surg Clin N Amer 1957; 37: 353.

3.- Witlin AG, Sibai BM: Magnesium sulphate therapy in preeclampsia and eclampsia. Obstet Gynecol 1998; 92 (5): 883-9.

4.- Lucas MJ, Leveno KJ, Cunningham FG: A comparison of magnesium sulphate with phenytoin for the prevention of eclampsia. N Engl J Med 1995; 333: 201-5.

5.- The Eclampsia Trial Collaborative Group: Which anticonvulsant for woman with eclampsia? Evidence 
from the collaborative eclampsia trial. Lancet 1995; 345: 1455-63.

6.- Duley L, Henderson-Smart D: Magnesium sulphate versus phenytoin for eclampsia. Cochrane Database Syst Rev 2003; CD000128.

7.- Belfort MA, Anthony J, Saade GR, Allen JC Jr: A comparison of magnesium sulfate and nimodipine for the prevention of eclampsia. N Engl J Med 2003; 348:304.

8.- Pryde G, Besinger R, Gianopoulos J, Mittendorf R: Adverse and beneficial effects of tocolytic therapy. Sem Perinat 2001; 25 (5): 316-40.

9.- Ramsey P, Rouse D: Magnesium sulphate as a tocolytic agent. Sem Perinat 2001; 25 (4): 236-47.

10.- Kasper DL, Braunwald E, Fauci AS, Hauser SI, Longo $D L$, Jameson JL, editors: Harrison's Principles of Internal Medicine. Part XIV Endocrinology and Metabolism: Bone and Mineral Metabolism. $16^{\text {th }}$ edition, McGraw-Hill 2005.

11.- Roberts JM: Magnesium for preeclampsia and eclampsia. N Engl J Med 1995; 333: 250.

12.- Manorot M, Tongsong T, Khettglang T: A comparison of serum magnesium sulphate levels in pregnant women with severe preeclampsia between intravenous and intramuscular magnesium sulphate regimens: a randomized controlled trial. J Med Assoc Thai 1996; 79 (2): 76-82.

13.- Hall DR, Odendaal HJ, Smith M: Is the prophylactic administration of magnesium sulphate in women with pre-eclampsia indicated prior to labor. BJOG 2000; 107: 903.

14.- Sibai BM: Magnesium sulfate prophylaxis in preeclampsia: Lessons learned from recent trials. Am J Obstet Gynecol 2004; 190: 1520.

15.- ACOG practice bulletin: Diagnosis and management of preeclampsia and eclampsia. Number 33, January 2002. Obstet Gynecol 2002; 99: 159.

16.- Alexander JM, McIntire DD, Leveno KJ, Cunningham $F G$ : Selective magnesium sulfate prophylaxis for the prevention of eclampsia in women with gestational hypertension. Obstet Gynecol 2006; 108: 826.

17.- Cholst IN, Steinberg SF, Tropper PJ, et al: The influence of hypermagnesemia on serum calcium and parathyroid hormone levels in human subjects. N Engl J Med 1984; 310: 1221 .

18.- Elliott JP: Magnesium sulphate as a tocolytic agent. Am J Obstet Gynecol 1983; 147: 277-84.

19.- Ghia N, Spong CY, Starbuck VN, Scialli AR, Ghidini $A$ : Magnesium sulfate therapy affects attention and working memory in patients undergoing preterm labor. Am J Obstet Gynecol 200; 183: 940-4.

20.- Sibai, BM, Lipsitz, J, Anderson, GD, Dilts, PV Jr: Reassessment of intravenous $\mathrm{MgSO} 4$ therapy in preeclampsia-eclampsia. Obstet Gynecol 1981; 57: 199.

21.- Madden C, Owen J, Hauth JC: Magnesium tocolysis: Serum levels versus success. Am J Obstet Gynecol 1990; 162: 1177-80.

22.- Hallak M, Berry SM, Madincea F, Romero R, Evans $M I$, Cotton $D B$ : Fetal serum and amniotic fluid magnesium concentration with maternal treatment Obstet Gynecol 1993; 81: 185-8.

23.- Lipsitz PJ: The clinical and biochemical effects of excess magnesium in the new born. Pediatrics 1971; 47 (3): 501-9.

24.- Kamitomo M, Sameshima H, Ikenoue T, Nichibateke $M$ : Fetal cardiovascular function during prolonged magnesium sulfate tocolysis. J Perinat Med 2000; 28: 377-82.

25.- Leviton A, Paneth N, Susser M, et al: Maternal Receipt of Magnesium Sulfate Does Not Seem to Reduce the Risk of Neonatal With Matter Damage. Pediatrics 1997; 99 (4): e2.

26.- FineSmith $R B$, Roche $K$, Yellin $P B$, et al: Effect of magnesium sulfate of the development of cystic periventricular leukomalacia in preterm infants. Am J Perinatol 1997; 14 (5): 303-7.

27.- Nelson K: Magnesium sulfate and risk of cerebral palsy in very low-birth-weight infants. JAMA 1996; 276: 1843-4.

28.- Crowther C, Hiller J, Doyle L, Haslam R: Effect of magnesium sulfate given for neuroprotection before preterm birth: a randomized controlled trial. JAMA 2003; 290 (20): 2669-76.

29.- The Magpie Trial Collaborative Group: Do women with pre-eclampsia, and their babies, benefit from magnesium sulphate? The Magpie Trial: a randomizedplacebo controlled trial. The Lancet 2002; 359: 187790.

30.- Kimberlin DF, Hauth JC, Goldenberg RL, et al: The Effect of Maternal Magnesium Sulfate Therapy on Neonatal Morbility in $\leq 1000$ gram infants. Am J Perinatol 1998; 15: 635-41.

31.- Golzarian J, Scott HWJ, Richards WO: Hypermagnesemia-induced paralytic ileus. Dig Dis Scie 1994; 39: 1138-42.

32.- Riaz M, Porat R, Brodsky NL, Hurt H: The effects of maternal magnesium sulfate treatment on newborns: a prospective controlled study. J Perinat 1998; 18 (6): 449-54.

33.- Sokal MM, Koenigsberger MR, Rose JS, Berdon WE, Santulli TV: Neonatal hypermagnesemia and the meconium-plug syndrome. N Engl J Med 1972; 286: 823-5.

34.- Brand JM, Greer FR: Hypermagnesemia and inestinal perforation following antacid administration in a premature infant. Pediatrics 1990; 85: 121-4.

35.- Narchi H: Delayed intestinal transit and arrhytmias due to iatrogenic neonatal hypermagnesemia. Int Pediatr 2002; 17 (3): 154-5.

36.- Rash DK, Huber PA, Richardson CJ, L'Hommedieu CC, Nelson TI, Reddi R: Neurobehavioral effects of neonatal hypermagnesemia. J Pediatr 1982; 100: 2726.

37.- Mofenson HC, Caraccio TR: Magnesium Intoxication In A Neonate From Oral Magnesium Hydroxide Laxative. Clinical Toxicology 1991; 29 (2): 215-22.

38.- Brady JP, Williams HC: Magnesium Intoxication in a Premature Infant. Pediatrics 1967; 40: 100-3. 